\title{
El estudio de la participación digital en la cultura. Hacia una propuesta analítica para
} el escenario cubano*

\author{
Studying the Digital Participation in the Culture. Towards an Analytical Proposal for the Cuban Scenario \\ O estudo da participação digital na cultura. Rumo a uma proposta analítica para o cenário cubano
}

Hamlet López García ${ }^{a}$

Instituto Cubano de Investigación Cultural "Juan

Marinello", Cuba

hamlet.lopez@cubarte.cult.cu

ORCID: http://orcid.org/0000-0001-8625-0374

DOI: https://doi.org/10.11144/Javeriana.syp37-72.epdc

Redalyc: http://www.redalyc.org/articulo.oa?id=86057225004

Fecha de recepción: 19 Abril 2017

Fecha de aprobación: 12 Octubre 2017

\section{Resumen:}

El presente artículo pretende contribuir a la comprensión de la participación digital en la cultura del entorno cubano. Para ello se exponen y analizan los principales resultados del estudio cualitativo, por medio de la Teoría Fundada, una experiencia concreta de participación en Cuba: una comunidad de software libre. Los resultados de la investigación, permiten conceptualizar a la participación digital en la cultura tal y como ocurre en Cuba, como un proceso fuertemente contextualizado, dependiente del entorno institucional en el que se desenvuelve. Esta participación digital permite una comprensión en la cultura más integral y productiva, desde el punto de vista teórico y conceptual.

Palabras clave: participación digital en la cultura, software libre, cultura digital.

\section{Abstract:}

This works seeks to contribute to the understanding of the digital participation in the culture in the Cuban scenario. To do so, the main outcomes from a qualitative study on a concrete participation experience in Cuba are displayed and analyzed based on the Grounded Theory: a free software community. The research results allow to conceptualize the digital participation in the culture just in the way they occur in Cuba, i.e., a strongly contextualized process depending on the institutional environment where it is being developed. This digital participation enables a more comprehensive and productive understanding of the culture under a theoretical and conceptual view.

Keywords: digital participation in the culture, free software, digital culture.

\section{Resumo:}

O presente artigo visa contribuir à compreensão da participação digital na cultura do ambiente cubano. Para isso, expõem-se e analisam-se os principais resultados do estudo qualitativo, por meio da Teoria Fundada, uma experiência concreta de participação em Cuba: uma comunidade de software livre. Os resultados da pesquisa permitem conceituar a participação digital na cultura tal e como acontece em Cuba, como um processo fortemente contextualizado, dependente do contexto institucional no que está se desenvolvendo. Essa participação digital permite uma compreensão na cultura mais integral e produtiva, desde o ponto de vista teórico e conceitual.

Palavras-chave: participação digital na cultura, software livre, cultura digital.

\section{Introducción}

En Cuba, como en otras sociedades, son visibles los efectos culturales y comunicacionales de la apropiación social de las tecnologías digitales tanto para la información como para la comunicación. Se puede constatar a nivel anecdótico, una mayor capacidad para la participación de los miembros de la sociedad en la producción, circulación y consumo de contenidos culturales impulsada por las tecnologías digitales, esta participación se da muchas veces de manera inédita y emergente. La estructura de poder que sostiene la circulación de recursos Notas de autor:

\footnotetext{
autor de correspondencia. Correo electrónico: hamlet.lopez@cubarte.cult.cu
} 
simbólicos en el campo cultural cubano, se halla bajo fuertes presiones, por la entrada de nuevos actores que actúan como productores y distribuidores de bienes y servicios culturales. Pero a pesar de las semejanzas con el momento de cambio cultural a nivel internacional, es difícil pensarlo desde Cuba a través de los marcos conceptuales dominantes en la literatura al uso.

En los conceptos teóricos, prevalecientes en el campo de los estudios culturales, los media studies y la comunicación social, hay una dependencia no siempre manifiesta al contexto de sus autores, dando por sentado un capitalismo altamente informatizado. La fuerte presencia de las industrias culturales, las sinergias entre el creciente acceso a la banda ancha y plataformas sociales como Youtube o Facebook además de un régimen fuerte de defensa de la propiedad intelectual, funcionan en esa literatura como presupuestos de las principales construcciones conceptuales. En Cuba, en virtud de su particular organización económica y política, simplemente no tiene sentido tomarlos como supuestos para una reflexión sobre la participación a través de las tecnologías digitales.

La tesis principal del artículo consiste en si se quiere entender la democratización en el consumo, distribución junto con la producción de contenidos y servicios culturales, gracias a las tecnologías digitales, como un fenómeno presente también en Cuba. Se necesita una conceptualización de la participación digital en la cultura, con un carácter más dependiente del contexto que la encontrada usualmente en la literatura disponible. Para ello, en lugar de usar un marco de análisis ya establecido, este se va a construir con ayuda del estudio cualitativo de una experiencia concreta de participación digital en la cultura: una comunidad cubana de software libre.

\section{Enfoques conceptuales para el estudio de la participaciónón digital ¿Algúnún traje a la medida?}

Cristopher M. Kelty (2013), en un artículo cuya capacidad crítica de mirada al campo propio no es común encontrar dentro de los media studies, relata cómo han proliferado los términos para dar cuenta de los efectos de internet y los nuevos medios sobre la participación, sin que esos términos dialoguen unos con otros. La 'producción entre pares', de Yochai Benkler; produsage, de Axel Bruns; 'cultura participativa' y 'cultura convergente', de H. Jenkins; y otros más como wikinomics, 'sabiduría de las multitudes' o "públicos recursivos”, a pesar de referirse a fenómenos semejantes y con aspectos coincidentes, son tratados como entes conceptuales aislados y originales, sin antecedentes y no traducibles entre sí. Aún más grave, para Kelty, es la ausencia de una reflexión sistemática y epistémica del término 'participación', la cual ata las definiciones generadas a manifestaciones concretas de participación en la cultura digital y por su escaso nivel de abstracción limita la comparación entre sí de las diversas manifestaciones.

Henry (Jenkins \& Couldry, 2014) comparte el malestar con el poco tratamiento del concepto dentro de su campo de estudio, y pide mayor riqueza de matices en los análisis de lo que ocurre en cada momento y locación particular de participación. Para Jenkins, se necesitan nuevas formas de teorización y una actitud un poco más escéptica sin llegar a ser cínica, alejada por igual de los extremos de la utopía y la distopía cibernética. Nick Couldry, en el mismo artículo comparte ese malestar y apunta al carácter situado de los discursos sobre la participación. De manera sumamente interesante, introduce una perspectiva geopolítica, cuando hace notar la capacidad de algunos de esos discursos de volverse hegemónicos, acallar otras maneras de hablar y pensar acerca de los propósitos de la participación así como sus medios.

La ausencia principal es una elaboración explícitamente más dependiente del contexto, de lo que es 'participación'. Como dice Kelty en la conclusión de su artículo (Kelty, 2013), si existen diferentes tipos de participación, entonces deben ser explicadas pensando específicamente en sus prácticas, herramientas, ideologías y tecnologías que las hacen posibles. La reflexión sobre participación debe incorporar también a sus estructuras, el proceso de gobernanza e inclusión, la infraestructura del software, redes, protocolos así 
como la retórica y expectaciones de los individuos. A esto, cabe añadir que también es necesario considerar las sinergias que se dan entre las diferentes tecnologías digitales presentes en la sociedad, entre estas e igualmente el entorno institucional y legal. En el caso cubano, esto último resulta mucho más evidente. Cuba es una sociedad no capitalista, donde el mercado no es el principal mecanismo para la distribución de bienes y servicios a la población, la penetración de las tecnologías digitales es muy baja y además el Estado tiene un lugar predominante en la vida social y económica. Cualquier experiencia participativa cubana, necesariamente se apoya, tiene en cuenta o choca con algún elemento estatal.

En la definición de Henry Jenkins de la cultura participativa (Jenkins, 2006) (Jenkins, Purushotma, Clinton, \& Robison, 2009) es muy difícil darle algún lugar al elemento institucional, y además está muy vinculada a los públicos de las industrias culturales. Las elaboraciones de Henry Jenkins, tienen en su base el impulso por comprender el fuerte compromiso y actividad de los aficionados alrededor de franquicias audiovisuales como "Star Trek" o "Stars Wars". Para ello, toma como base dos elementos estructurales: la comunidad como formación social que da soporte y sirve de plataforma a la producción y circulación de contenidos; y como segundo elemento estructural la tecnología digital, que disminuye las barreras tanto económicas así como tecnológicas para el involucramiento de nuevos sectores sociales en la participación cultural. Es así como construye su concepto de participación cultural, presentándolo como una nueva forma de producción cultural, llevada a cabo por individuos organizados en comunidades que poseen:

- Relativamente pocas barreras para la expresión artística y el compromiso cívico.

- Con fuerte soporte entre los miembros de la comunidad para crear y compartir las creaciones

- Con algún tipo de tutoría informal de los miembros más experimentados hacia los novicios.

- Donde los miembros de la comunidad creen que sus contribuciones importan y además

- Sienten que tienen algún grado de conexión social entre sí, al menos la conexión dada por el interés que la opinión sobre sus creaciones.

Por ello, su noción de lo que son los públicos, particularmente el público de las industrias culturales, se caracteriza como un ente activo, que conforma, comparte, remezcla y recontextualiza contenidos mediáticos en modos imprevistos, dentro de grandes comunidades virtuales. Jenkins, reconoce en el público un deseo de interacción, de alimentar lazos sociales y de construir comunidades aún más grandes, motivado tanto por su fuerte relación con los contenidos mediáticos, como por las facilidades que las tecnologías digitales ponen a su alcance. Para él, la incorporación que hace el público de sus experiencias y sentidos a los contenidos mediáticos que circulan por la red, es una participación no solo legítima, sino empoderadora (Jenkins, 2013).

La definición brindada por Axel Bruns (2008) también presupone la actividad de industrias culturales fuertes, al punto de que tiene en cuenta en su análisis las estrategias de las corporaciones mediáticas para integrar las producciones de los usuarios dentro de sus estrategias comerciales, algo que él denomina "cosechar la colmena", y que identifica como uno de los riesgos que enfrenta la actividad de los produsuarios.

Como último ejemplo, se puede citar al investigador y profesor radicado en Holanda Tobias Shafer para hablar de la democratización en la producción, distribución, consumo de contenidos y servicios culturales basado en el concepto de "industria cultural extendida", o lo que es lo mismo, la extensión de los modos de producción capitalistas a las esferas de la vida privada de los usuarios que participan en la creación y uso de contenidos y artefactos digitales (Schäfer, 2011).

No obstante las dificultades conceptuales y empíricas para encontrar en Cuba fuertes industrias culturales mediáticas y sus públicos fidelizados, estamos dispuestos a reconocer intuitivamente procesos emergentes de participación digital socio cultural si a partir de apropiaciones inesperadas de las tecnologías digitales, aumenta la cantidad de personas que producen y distribuyen contenidos. En el caso cubano, se alude a que estamos en presencia de procesos participativos en la cultura digital cuando circulan contenidos audiovisuales por fuera del monopolio estatal sobre la distribución audiovisual, cuando se construyen redes de telecomunicación de manera autogestionaria por los habitantes de los diferentes barrios de la Ciudad de la 
Habana, para jugar en línea e intercambiar datos, y cuando surgen comunidades de usuarios de software libre que sobreviven en un entorno con baja presencia de las tecnologías digitales en la sociedad y estrecho control estatal sobre los servidores web de acceso público.

En Cuba la circulación de contenidos audiovisuales por fuera del monopolio estatal sobre la producción y distribución audiovisual, a manera de distribución reticular vía interfaz USB y soportes físicos, como los discos duros externos o memorias flash, tiene su mejor ejemplo en el denominado 'paquete', una compilación digital que abarca contenidos audiovisuales, informáticos, musicales y de libros, además de noticias, todos digitalizados, provenientes de las principales industrias creativas del mundo, la mayoría descargados ilegalmente y organizados temáticamente por carpetas, que ocupa de $500 \mathrm{gb}$ a un terabyte, a la que se le añaden anuncios comerciales de pequeños negocios cubanos y que se distribuye una vez a la semana copiándose de disco duro a disco duro, sea portátil o interno. Según algunos testimonios, puede estimarse que su distribución siempre de copia en copia, alcanza a todo el país. Ofrecen una amplia variedad de productos provenientes de diversos países: Estados Unidos, pero también México, España, India, Francia, entre otros y se está convirtiendo en uno de los principales canales para la publicidad de los emergentes negocios privados cubanos. Si bien puede estudiarse como uno de los casos de los denominados "Media Movile", como lo hace la autora Ana Cristina Pertierra (Pertierra, 2012), aquí prefiero destacar su carácter reticular, no lineal, a veces basado en su copia a cambio de dinero, pero también distribuido a través de redes de amigos o conocidos de manera gratuita (Rodríguez et al., 2016).

Las redes de telecomunicación comunitarias se basan en la capacidad que tienen las computadoras para comunicarse entre sí. El ejemplo más notorio y consolidado de estas redes comunitarias es la llamada Snet (Figueredo \& Dominguez, 2016), que según algunos testimonios, alcanza prácticamente la totalidad de la ciudad de La Habana. Se puede considerar la SNet como una respuesta auto gestionada desde algunos sectores de la sociedad -con la capacidad y los recursos para ello- a la contradicción entre la lentitud en el despliegue de las redes públicas de datos para los ciudadanos por parte de las instituciones estatales, la imposibilidad legal para la población de establecer de manera autónoma, redes de computadoras que ofrezcan servicios y la evidente naturalidad de las tecnologías digitales tanto propiciar como para facilitar el trabajo en red. Dichas redes, creadas inicialmente para jugar online o compartir ficheros, con el tiempo han ido creciendo en servicios, hasta el punto de hospedar redes sociales, sitios de microbloging, y múltiples foros, recreando en pequeño la intensa dinámica social de intercambio online que se tendría con el acceso pleno a internet. La implementación de las redes informáticas comunitarias al margen de lo que permite la ley —aunque toleradas hasta cierto punto-, es vivida por sus protagonistas como algo inevitable, dadas las crecientes facilidades que ofrece la tecnología para la interconexión y la vida en red, en una especie de tecno determinismo interesado e ingenuo, que oculta en realidad dinámicas sociales más profundas y por ello, mucho más determinantes de la generalización sobre las prácticas de compartir online, comunicar y jugar, las cuales traen consigo las generaciones más recientes, soportadas, eso sí, en una creciente oportunidad para hacerlo gracias a la tecnología.

Por último, la comunidad cubana de software libre nace de la organización espontánea de un grupo de usuarios de tecnologías libres. Ante la incapacidad de recibir información y descargar software de los repositorios de software internacionales, debido a las pobres condiciones de conectividad de la isla, deciden organizarse como comunidad virtual y brindar soporte a partir de sus propios conocimientos, compartiendo entre sí y enriqueciendo colaborativamente el conocimiento sobre Linux y otras tecnologías libres, a través de una lista de correo, un foro virtual y una wiki. Lo llamativo de la comunidad cubana, es que no pueden administrar directamente el servidor web donde alojan sus servicios, por el marco legal cubano que hasta hace muy poco no permitía que las personas naturales contrataran nombres de dominio ni publicaran servicios web. Otra particularidad es el uso de las escasas conexiones a internet entre sus miembros, las cuales son puestas en común para la descarga de software y tutoriales (López, 2014). 
Cabe notar que para ninguno de los tres ejemplos mencionados, resulta vital ni la banda ancha, ni una fuerte presencia de las industrias culturales ni las plataformas de redes sociales como punto de partida. De hecho una característica notable de los dos primeros, resulta ser la circulación de contenidos audiovisuales y las redes informáticas, así como el proceso de construcción colectiva desde cero de una infraestructura para la participación. Tampoco la propiedad intelectual representa una coordenada fundamental para situar las actividades de los usuarios. La ausencia de relaciones económicas entre Estados Unidos y Cuba, sitúa a estas actividades en una especie de espacio protegido donde virtualmente no es relevante la propiedad intelectual, la circulación de contenidos audiovisuales y jugar videojuegos en línea se regula, mediante la potencial conflictividad política de los contenidos. Por la misma razón, las industrias culturales mayormente extranjeras, tienen un estatus de meros proveedores de contenidos, sin que determinen ritmos ni espacios para la circulación de los contenidos, y tampoco puedan resultar beneficiados de su consumo.

Dadas estas dificultades conceptuales, con el fin de observar en mayor detalle las particularidades de la participación digital en la cultura tal y como se da en el entorno cubano, se emprendió un estudio cualitativo de una experiencia de participación en particular: la comunidad cubana de software libre. El objetivo principal del estudio, consistió en encontrar dimensiones e indicadores relevantes para el análisis de dichas experiencias que en un momento posterior, fueran de utilidad para construir una propuesta teórico conceptual de la participación digital en la cultura que estuviera contextualizada en el escenario cubano. El estudio, se centró en el análisis de contenido de la lista de correos de la comunidad de software libre Grupo de Usuarios de Tecnologías Libres (GUTL), bajo el supuesto que los principales elementos que median la práctica participativa y que configuran su contenido, tendrían expresión en esa lista. A continuación, se presenta la comunidad cubana, se reseña brevemente el proceder metodológico y por último se muestran las principales categorías encontradas.

\section{La comunidad cubana de software libre GUTL}

Grupo de Usuarios en Tecnologías Libres [GUTL] (s.f.) es el nombre de un grupo social informal interesado en el software libre y abierto que organiza diversas actividades para la promoción de este tipo de software, tales como lo son festivales de instalación, conferencias, administración de una comunidad virtual basada en un Foro en línea, una lista de correos y una wiki. Vale recordar que el software libre es el conjunto de programas informáticos que en virtud de la licencia con que son publicados pueden ser copiados, estudiados, modificados, utilizados libremente con cualquier fin y redistribuido. La comunidad GUTL (s.f.), surgió de una reunión realizada el 13 de junio de 2009 a partir del ya existente Grupo de Usuarios de GNU/Linux Habana mediante una organización flexible. En aquel momento fueron propuestos como objetivos:

- Agrupar a todos los especialistas nacionales cubanos en materias asociadas al desarrollo de la sociedad de la información con tecnologías libres, dígase: software libre, hardware libre y conocimiento libre.

- Apoyar al Estado cubano en la creación de una red social de especialistas que puedan ayudar a todas las regiones del país, mediante la asesoría, en la toma de decisiones para alcanzar la soberanía e independencia tecnológica.

- Crear una entidad social, mediante el cual pueda ser ejercida a través de pago, o carente de ella la capacitación del personal en todas las regiones de la república de Cuba en las materias que confieren la transferencia tecnológica a la población cubana para alcanzar la migración del país a tecnologías libres.

- Organizar, financiar y colaborar en las ideas fundamentales para el desarrollo de software libre con fines sociales, no excluyendo la exportación, para la República de Cuba. 
Hasta enero del 2015, fecha en la que cerró la recogida de datos, la comunidad contaba con alrededor de 725 miembros que al menos habían enviado un correo a la lista, siendo este el servicio que cuenta con la mayor actividad.

\section{Metodología}

Después de una presentación de la investigación a la comunidad, que incluía una solicitud de colaboración y la posibilidad para quien quisiera en caso de no ser incluido en el estudio. Se descargaron los correos disponibles públicamente que circularon desde el 25 de mayo del 2012 hasta el 28 de enero del 2015. Se anonimizaron y extrajeron metadatos como fecha de envío, asunto del correo y cuerpo del correo. Estos últimos fueron usados como principales fuentes de datos. Dado que el corpus contenía un número considerable de correos — 25\#69 correos electrónicos - se aplicaron técnicas provenientes de la minería de textos, para reducir el volumen de información a tratar.

La Minería de Textos, combina técnicas de la minería de datos, recuperación de información y procesamiento del lenguaje natural entre otros campos para extraer, descubrir y vincular datos de grandes cuerpos de datos textuales (Feldman \& Sanger, 2007). En el caso de la presente investigación, se acudió a la minería de textos para guiar el proceso de recolección de datos que sirviera, para desarrollar teoría según el paradigma de Teoría Fundada (Glasser \& Strauss, 1967; Flick, 2009) y seleccionar los correos más relevante para las categorías emergentes del análisis. Este proceso iterativo, denominado muestro teórico según Uwe Flick, implica que las decisiones acerca de la elección de material empírico se realizan en el propio proceso de recoger e interpretar la información.

De los correos descargados, se extrajo una lista de todos los asuntos de los correos de la lista y la cantidad de correos agrupados bajo un mismo asunto. Esto permitió identificar aquellos correos que motivaron el mayor número de participación de los miembros de GUTL. Los asuntos con más de diez correos agrupados, fueron entonces clasificados inicialmente en grupos: Ayuda y soporte técnicos, que implica el traspaso de información técnica de cómo usar un software o adaptarlo para su uso; Vida de la comunidad, sobre la organización de actividades de la comunidad, normas y valores, dificultades de los miembros y de la comunidad;; y por último solicitud de ayuda no directamente relacionada con conocimientos técnicos, por ejemplo miembros preguntando en la lista quién tenía un determinado software. Los correos agrupados bajo los asuntos relacionados con temas de soporte técnico, fueron descartados inicialmente, y se leyeron aquellos correos con asuntos relacionados con la vida de la comunidad y ayuda no técnica y que tuvieran mayor número de correos agrupados bajo un mismo asunto, para extraer términos de búsqueda relacionadas con la temática de la investigación.

$\mathrm{Al}$ mismo tiempo los correos fueron transformados a texto plano e indexados en un motor de búsqueda para escritorio de código abierto, DocFetcher (http://docfetcher.sourceforge.net/), basado en Lucene, un conjunto de programas informáticos de código abierto para la recuperación de información. Este motor de búsqueda indexa los documentos a partir del valor tf-idf (Term frequency - inverse document frequency) que permite balancear el peso de aquellas palabras que son generalmente más comunes que otras. El indexado de los correos tuvo como objetivo poder recuperar los correos más relevantes según el término de búsqueda introducido.

Mientras que el primer listado de términos de búsqueda fue fijo, la recuperación y lectura de los correos más relevantes así como sus asuntos, constituyó un proceso iterativo de identificación de nuevos términos de búsqueda, que permitió completar la información e ir refinando las categorías iniciales, hasta alcanzar un estado de saturación teórica. Las categorías finalmente construidas fueron cuatro: tecnológica, competencial, constitutiva e institucional. Estas categorías se presentan brevemente a continuación. 


\section{Resultados}

\section{A) Categoría tecnológica}

La infraestructura tecnológica, está presente en la práctica participativa digital en la cultura en varias instancias. En el caso de la comunidad cubana de software libre, una primera instancia es el de su expresión real. Los productos y resultados de la participación en la comunidad, se ven mediados por el predominio de las conexiones estatales por sobre las domésticas tanto a internet como a cualquier otro tipo de redes. La segunda instancia, está relacionada con la escasa conexión a internet, la cual está en la base de un gran volumen de correos que circulan en la lista de correos, solicitando descargas o compartir por medios físicos información previamente descargada. Una tercera instancia, está en las cualidades funcionales de las tecnologías disponibles para la comunidad: el correo electrónico, el foro virtual, el ftp, pero también los soportes ópticos como el CD, DVD y USB para la copia de grandes volúmenes de información. Estos dispositivos, permiten compensar en parte el escaso nivel de penetración de las tecnologías digitales en la sociedad cubana, pero también imponen limitaciones a la circulación de contenidos, y por tanto dejan su huella en la naturaleza de la actividad participativa.

De esta manera la comunidad de GUTL, no solo funciona como una comunidad para la promoción del software libre, sino también como un mecanismo de socialización de los recursos escasos de conectividad entre todos los miembros.

La ausencia de conectividad, también está en la raíz de otra característica distintiva de la comunidad cubana de software libre: Las numerosas copias locales de repositorios de software libre, tanto en instituciones como en las casas de los participantes.

El peso de las cualidades de la tecnología disponible, también es observable en las prácticas de la comunidad cubana de software libre. Principalmente en el uso del correo electrónico, gracias al cual se comparte información y vínculos para descargar, pero que es empujado al límite de sus capacidades de almacenaje, al punto de usarlo para compartir archivos muy grandes para las capacidades del correo, subdividiéndolo en pedazos más pequeños:

La infraestructura tecnológica, marca los ritmos y capacidades de la comunidad GUTL de participar digitalmente en la promoción de la cultura basada en el software libre. Ya sea por el peso que tienen las redes de telecomunicación estatales para sostener el trabajo de la comunidad, la escasa conexión a internet al igual que a otras redes, así como el aprovechamiento de las cualidades funcionales de copia y almacenamiento de información.

\section{B) Categoría competencial}

Los miembros de la comunidad GUTL, al presentarse como una comunidad de soporte al software libre, de por sí exhiben niveles altos de competencia tecnológica. De la misma manera, aunque no todos tienen conocimientos de programación, muestran disposición a aprender, compartir y reutilizar los códigos que han desarrollado para facilitar sus tareas. Así mismo tienen los conocimientos para instalar y gestionar vías digitales para la circulación de contenidos. Por último, también pueden localizar, compartir y consumir contenidos afines a sus intereses. 


\section{C) Categoría constitutiva}

Los miembros de la comunidad, muestran niveles elevados de participación digital. No solo han personalizado herramientas para mantener los servicios de GUTL, la lista de correo, el foro virtual y otras herramientas como plataformas para la participación digital. Sino que también muestran un sentido del potencial impacto que tienen las tecnologías digitales libres para la sociedad cubana, por lo que intentan una promoción más activa incluso a nivel jurídico. Con ella, manifiestan una percepción de la naturaleza social de las tecnologías y de su capacidad para estimular o inhibir la participación del resto de la sociedad.

Los sentidos de la práctica participativa dentro de la comunidad GUTL se expresan en dos instancias: una primera instancia relacionada con la socialización de conocimientos y tecnologías entre los participantes de la comunidad, la segunda instancia ubica la promoción del software libre en la sociedad como uno de las razones de ser de la comunidad, articulándose inclusive con un proyecto político.

La historia compartida de la comunidad GUTL en especial su relación con la institucionalidad cubana, de la manera en que es recordada y recontada a los miembros nuevos que llegan, también tiene un peso en las normas y en la manera de gestionar los servicios que se ofrecen, así como en la difícil convivencia de los participación digital con la institucionalidad. Así las experiencias compartidas se formalizan en reglas de lo que se puede y no se puede hablar en la lista, además de la experiencia de cómo debían manejar la lista de correo, el foro virtual y cualquier otro servicio que quisieran implementar.

\section{D) Categoria institucional}

La institucionalidad está presente en la actividad de la comunidad GUTL en cuatro instancias principales: la primera es en el soporte para los servicios, la comunidad brinda sus servicios a través de la infraestructura tecnológica de una institución estatal. La segunda es el ordenamiento jurídico cubano, el cual solo permite a las personas jurídicas publicar servicios web y administrar servidores, lo que obliga a plantearse un objetivo adicional a la comunidad, la de convertirse en una personalidad jurídica para así poder administrar sus servicios sin depender de una institución externa. La tercera instancia de la dimensión institucional, es lo que se puede denominar como la regulación por medio de políticas internacionales de la circulación de bienes y contenidos digitales. En el caso cubano se refiere específicamente al conjunto de leyes y regulaciones del gobierno norteamericano y que instrumentan el bloqueo económico y comercial a Cuba desde 1961. Este bloqueo, busca impedir la difusión a Cuba de tecnologías informáticas, incluyendo las que están cubiertas por las licencias Copyleft. La cuarta y última instancia lo constituye la existencia de las licencias de derecho de autor tipo copyleft, que regulan y hacen posible la existencia de comunidades de software libre como la de GUTL.

\section{Conclusiones}

La comunidad cubana de software libre, tiene la potencialidad compartida con otras comunidades similares a nivel internacional, de desarrollar altos niveles de participación, que incluye no solo la creación de tecnología sino también el activismo en la promoción de la cultura libre y abierta, al igual que el sostenimiento de los bienes comunes culturales. Sin embargo el marco legal e institucional en que tiene que desplegar sus prácticas le hacen adquirir características inéditas frente a otras comunidades. Una de ellas, es la imposibilidad de administrar directamente los servidores donde están hospedados sus principales servicios en línea, como la lista de correos. Tienen que depender de una institución estatal con personalidad jurídica y por tanto con capacidad legal, según el marco jurídico cubano, para poseer y administrar servidores web. Además las dificultades para la descarga de software y otros contenidos de internet en Cuba - por dificultades de infraestructura pero también por efectos del bloqueo norteamericano a Cuba que aún es vigente- ralentizan 
la velocidad de la difusión de nuevas aplicaciones de software libre o tutoriales. Estas dependen en gran medida de las redes personales para intercambiar los ficheros a través de discos duros externos, memorias flash u otros dispositivos físicos.

El Estado cubano tiene un importante papel en la regulación de la circulación y consumo de los contenidos y servicios digitales. Este papel central del Estado, que a su vez es la garantía de un acceso democrático y soberano a la cultura y uno de los pilares de los altos niveles educativos de la población cubana, se instrumentaliza por medio de la propiedad efectiva sobre los medios de comunicación y sus recursos. En el escenario de las tecnologías digitales, lo anterior significa la propiedad estatal sobre las redes de telecomunicación, puntos de acceso a la red, proveedores de servicios de internet, servidores web públicos y los servidores de nombres de dominio.

Por ejemplo si se analiza la mayoría de las resoluciones y legislaciones cubanas vigentes hasta enero del 2015 sobre el tema de las telecomunicaciones y servicios web (Silva, 2014), se puede observar que un usuario puede participar en el uso de contenidos y servicios ya sea como persona natural o como persona jurídica. Ambas figuras pueden hacer un contrato con un proveedor de internet o ser autorizados por un proveedor de internet en una red privada. Sin embargo, para participar como productor de contenidos y servicios o como mediador - es decir, como gestor de una red propia de telecomunicación-, solo las personas jurídicas están capacitadas por la ley.

En la literatura sobre la participación digital, especialmente la que proviene de los Estudios Culturales, suele predominar la mención a la mayor democratización en la producción, circulación de contenidos, servicios culturales y el desafío consiguiente al poder tanto de los productores como distribuidores tradicionales (Delwiche \& Henderson, 2010). Estos son elementos que se pueden encontrar en las experiencias cubanas de participación digital en la cultura, sin embargo, otras dimensiones de las relaciones entre regulación del comportamiento y tecnologías que destacan en el caso cubano, no suelen ser abordadas e incluso han quedado ofuscadas bajo un alud de literatura celebratoria sobre las redes sociales como Twitter, YouTube o Facebook y lo que los sujetos hacen en ellas. Por ejemplo una dimensión importante pero difícil de encontrar, es el rol de los poderes públicos que al trazar políticas para la cultura, la innovación, la educación y la ciencia, tienen un impacto directo en las capacidades para aprovechar las capacidades de las tecnologías en las prácticas culturales. Otra dimensión también con poca presencia, es el poder de determinar desde la tecnología quién participa, quién no y cómo se hace. Ambos expresan instancias específicas de regulación que configuran el proceso de participación y establecen fracturas en la apropiación social de la tecnología. Por lo mismo, estas son dimensiones que se intuyen decisivas si se quiere entender el paisaje completo de la participación digital en la cultura, un paisaje que integra a Wikipedia junto a Facebook, las comunidades de software libre y las iniciativas comunitarias de redes inalámbricas.

Estas ausencias resultan especialmente llamativas si se piensa que en el estudio de la participación digital en la cultura se choca inevitablemente con la relación entre la apropiación de la tecnología y las capacidades de agencia del sujeto, y cómo ésta es regulada para determinar lo que se puede y no se puede hacer. A juzgar por autores de mucho peso como los ya citados Henry Jenkins (Jenkins, Ford, \& Green, 2013) o Axel Bruns (2013a) las manifestaciones de participación digital, como las publicaciones en Twitter o en Facebook, o la publicación de videos amateurs gracias a Youtube operan en un vacío institucional, auto generadas y auto explicativas, si acaso conectadas con la genialidad de las empresas tecnológicas, sus innovaciones y el fervor de los públicos aficionados. Esta visión necesariamente limita las implicaciones sociales y políticas del estudio de la participación, su aplicabilidad, banaliza sus resultados e impide el diálogo con el resto de las disciplinas sociales. 


\section{Referencias}

Bruns, A. (2008). The Future Is User-Led: The Path towards Widespread Produsage. Fibreculture Journal, (11). FCJ-066 The Future Is User-Led: The Path towards Widespread Produsage. Recuperado el 25 de octubre de htt p://eleven.fibreculturejournal.org/fcj-066-the-future-is-user-led-the-path-towards-widespread-produsage/

Bruns, A. (2013a). From prosumption to produsage. In R. Towse \& C. Handke, (Eds.) Handbook on the Digital Creative Economy (p. 67 -78). Cheltenham: Edward Elgar Publishing.

Bruns, A. (2013b). Exploring the Pro-Am Interface between Production and Produsage. Frontiers in New Media Research, 15, 241-258.

Delwiche, A., \& Henderson, J. (Eds.). (2010). The Participatory Cultures Handbook. New York: Routledge.

Feldman, R., \& Sanger, J. (2007). The text mining handbook: advanced approaches in analyzing unstructured data. New York: Cambridge University Press.

Figueredo, O., \& Dominguez, L. E. (2016, September 16). SNet: La primera comunidad inalámbrica en Cuba, Canal USB. Recuperado October 16, 2016, de http://www.cubadebate.cu/noticias/2016/09/16/snet-la-primera-com unidad-inalambrica-en-cuba-fotos-infografia-y-pdf/

Flick, U. (2009). An introduction to qualitative reaearch. London: Sage Publications, Inc.

Glasser, B., \& Strauss, A. (1967). The discovery of grounded theory: Strategies for qualitative research. Chicago: Aldine.

Grupo de Usuarios en Tecnologías Libres [GUTL] (s.f.). Recuperado de http://gutl.jovenclub.cu

Jenkins, H. (2006). Fans, Bloggers and Gamers: Exploring Participatory Culture. New York: New York University Press.

Jenkins, H., Ford, S., \& Green, J. (2013). Spreadable media: Creating value and meaning in a networked culture. New York: New York University Press.

Jenkins, H., \& Couldry, N. (2014). Participations: Dialogues on the Participatory Promise of Contemporary Culture and Politics-Introduction. International Journal of Communication, 8, 1 - 6. Recuperado de http://ijoc.org/i ndex.php/ijoc/article/view/2748/1119

Jenkins, H., Purushotma, R., Clinton, K., \& Robison, A. (2009). Confronting the challenges of participatory culture: Media education for the 21st century. Cambridge: MIT Press.

Kelty, C. M. (2013). From participation to power. En Delwiche, A., \& Jenifer Jacob Henderson (Eds.). The participatory cultures handbook (pp. 22-31). New York: Routledge.

López, H. (2014). Las comunidades virtuales de software libre en Cuba. Difusión del conocimiento libre en un entorno de baja penetración de las tecnologías de la información. En B. Levy (Coord.) Bienes comunes. Espacio, conocimiento y propiedad intelectual (pp. 83-118). Buenos Aires: CLACSO.

Pertierra, A. C. (2012). If they show Prison Break in the United States on a Wednesday, by Thursday it is here: Mobile media networks in twenty-first-century Cuba. Television \& New Media, 13(5), 399-414. https://doi.org/10.1 $177 / 1527476412443564$

Rodríguez, F. A., López, M. M., Fournier, A. B., Puig, P. E. M., García, H. L., Petierra, A. C., ... Fernández, Y. A. (2016). Copia y comparte. Visiones sobre las prácticas de circulación y consumo de bienes culturales en entornos no institucionales en Cuba//Copy and share. Visions around practices circulation and consumption of cultural goods in non-institutional environments. Revista Cubana de Información Y Comunicación, 5(10), 143-170. Recuperado de http://www.alcance.uh.cu/index.php/RCIC/article/view/71/71

Schäfer, M. T. (2011). Bastard culture!: how userparticipation transforms cultural production. Amsterdam: Amsterdam University Press.

Silva, M. R. (2014). La hora de los desconectados : evaluación del diseño de la política de "acceso social" a internet en Cuba en un contexto de cambios.. Critica Y Emancipación, VI(11), 291-377. Recuperado de http://www.bibli oteca.clacso.edu.ar/ojs/index.php/critica/article/view/30 
Hamlet López García. El estudio de la participación digital en la cultura. Hacia una propuesta ...

\section{Notas}

* Artículo de investigación.

Licencia Creative Commons CC BY 4.0

Cómo citar: López, H. (2018). El estudio de la participación digital en la cultura. Hacia una propuesta analítica para el escenario cubano. Signo y Pensamiento, 37(72), 45-55. https://doi.org/10.11144/ Javeriana .syp37-72.epdc 